\title{
Axisymmetric Hopf bifurcation in a free surface rotating cylinder flow
}

\author{
$\begin{array}{lll}\text { S. J. Cogan } & \text { G. J. Sheard } & \text { K. Ryan } \\ & \end{array}$
}

(Received 13 August 2008; revised 24 October 2008)

\begin{abstract}
Using highly resolved simulations of the axisymmetric NavierStokes equations and a truncated Landau model we investigate the behavior of the flow in the vicinity of the axisymmetric Hopf-type transition in an open cylinder of height-to-radius aspect ratio of $3 / 2$. Rotating flows in open cylinders have many practical applications for which the knowledge of the different flow states encountered is of value. We report on the location and non-linear evolution characteristics of the bifurcation and present, for the first time, evidence that confirms that transition occurs via a non hysteretic supercritical Hopf bifurcation, and visualisations of the mode to fully define the transition.
\end{abstract}

\section{Contents}

1 Introduction

http://anziamj.austms.org.au/ojs/index.php/ANZIAMJ/article/view/1432 gives this article, (c) Austral. Mathematical Soc. 2008. Published November 7, 2008. ISSN 1446-8735. (Print two pages per sheet of paper.) 
2 Problem formulation

$\mathrm{C} 253$

2.1 Numerical details . . . . . . . . . . . . . . . . C254

2.2 Methodology .................... C255

2.3 The Landau equation . . . . . . . . . . . . . . . C256

3 Results

C257

4 Conclusion

C262

References

C263

\section{Introduction}

The transitions from a steady axisymmetric base state to an unsteady and/or three dimensional flow of the swirling fluid inside a cylinder have been much debated and the bifurcation properties of the flow in an enclosed cylinder are well documented $[1,3,4,7,9]$. However, the same cannot be said for the bifurcations of the flow in an open cylinder with a free surface. Few investigations into this configuration have been performed and knowledge of the stability and bifurcation characteristics of the system is patchy. Lopez [6] first looked at the transition of the steady axisymmetric base state to an unsteady axisymmetric state for an open cylinder with aspect ratio $\mathrm{H} / \mathrm{R}=$ 1.5 as part of a broader numerical investigation into the symmetry properties of the system. He concluded that there existed some hysteresis between the steady and unsteady branches, finding that while the unsteady flow state was generally observed from $\mathrm{Re}=2650$ and above, the steady flow state could be reached at Reynolds numbers as high as $R e=3200$ via manipulation of the initial conditions. Specifically, for a Reynolds number of say $R e=2675$, using the steady solution at $R e=2600$ as the initial condition caused the flow to evolve to the periodic branch. However, if the steady $R e=2650$ solution was employed as the initial condition for the $R e=2675$ case, the solution was found to converge to a steady state. Thus Lopez [6] was able 
to extend the steady solution to $\operatorname{Re} \sim 3200$, by making successively smaller increases in Re, and thereby concluded that the transition was hysteretic, having both stable steady and unsteady solutions at a number of points in the parameter space.

Brons et al. [2] investigated numerically the flow in an open cylinder over a wide range of aspect ratios, $0.25 \leq \mathrm{H} / \mathrm{R} \leq 4.0$, limiting the flow to a steady axisymmetric subspace to investigate the topological bifurcations of the system. They revealed a rich array of possible flow structures including some previously unidentified states, but their investigation was limited to bifurcations of a topological nature and did not focus on the stability of the flow as such. However, in order to ascertain the Reynolds number limits within which their analyses were valid they did investigate the stability limit for steady flow. They stated that for $\mathrm{H} / \mathrm{R}=1.5$ the steady flow became time-periodic at $\mathrm{Re}=2640$, agreeing to within half a percent with Lopez [6] on the location of the bifurcation. However the theory used to derive the location appeared to assume that the bifurcation was non hysteretic (as had been proven erstwhile was the case in an enclosed cylinder). This assumption was contrary to the finding of Lopez [6] whose results suggested that the transition was hysteretic by nature. It is this apparent discrepancy that we address in this article.

\section{Problem formulation}

The system being investigated is shown schematically in Figure 1(a). A vertically oriented circular cylinder with radius $R$ and height $H$ is filled with a fluid of kinematic viscosity $v$. The bottom is a rotating disk and the top is a flat stress-free surface. Lopez et al. [6, 8] give information regarding the modeling of this flow with a flat stress-free surface; it is shown that in 'deep' systems $(H / R \geq 1)$, and for low Froude numbers, this approximation is valid. The rotation of the base at a constant angular velocity $\Omega$ results in spin-up 
(a)

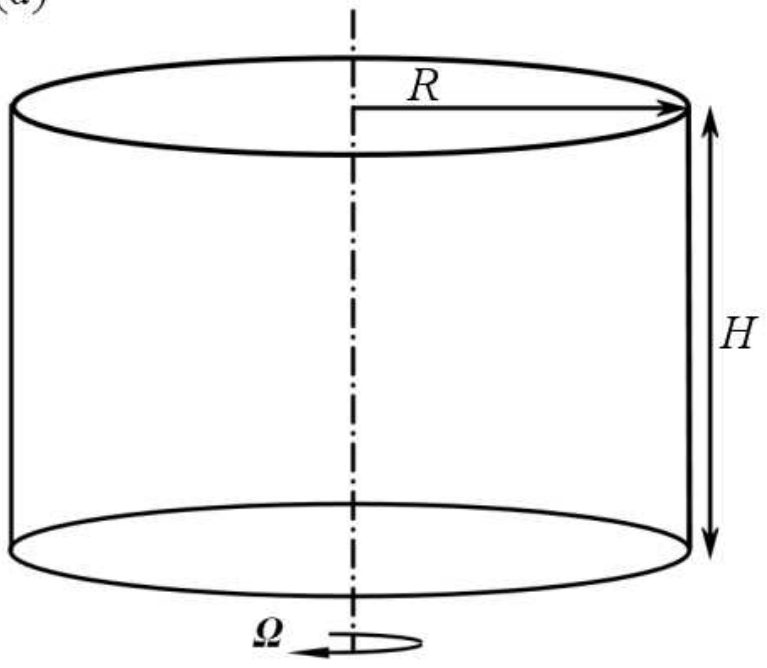

(b)

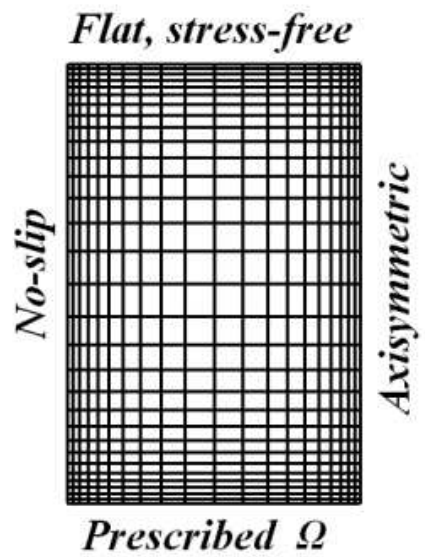

Figure 1: (a) Schematic of the flow scenario being considered, showing the key system parameters, and (b) the grid with macro elements and boundary conditions indicated. This represents the left half of the meridional plane.

of the fluid and the evolution of a non-trivial flow state in the meridional plane. The flow is fully defined by two parameters, the aspect ratio $H / R$ and the Reynolds number $R e=\Omega R^{2} / v$. Under the right combination of $H / R$ and Re a form of vortex breakdown known as the "vortex breakdown bubble" is known to occur [2].

\subsection{Numerical details}

An in house spectral element ( $\mathrm{SE}$ ) package is used to perform the computations $[11,12]$. The governing equations for the flow are the incompressible, 
unsteady momentum equation for a continuum,

$$
\frac{\partial \mathbf{u}}{\partial \mathrm{t}}+\mathbf{u} \cdot \nabla \mathbf{u}=-\nabla p+v \nabla^{2} \mathbf{u}
$$

together with the continuity equation

$$
\nabla \cdot \mathbf{u}=0
$$

where $\mathbf{u} \equiv\left(\mathbf{u}_{r}, \mathbf{u}_{z}, \boldsymbol{u}_{\theta}\right)(r, z, t)$ is the axisymmetric velocity vector (independent of the azimuthal coordinate), $t$ is time and $p$ is the kinematic static pressure. The flow is computed in the meridional semi-plane, which is discretised into a mesh of quadrilateral elements. Within each element high order polynomial basis functions are employed to approximate the flow variables over the Gauss-Legendre-Lobatto quadrature points. This permits efficient computations and the exponential spatial convergence for which SE methods are known. For time integration a third order, backwards multistep scheme is used [5].

The domain with the sparse macro-element mesh is shown in Figure 1(b). A P-type resolution study was performed on the base mesh revealing that polynomial basis functions of order $P=9$ adequately resolved the flow in the thin Ekman boundary layer at the base and the complex flow in the interior, at reasonable computational expense. A time step of $\delta t=0.005$ was chosen to satisfy both temporal accuracy and the stability requirements of the code.

\subsection{Methodology}

The computations are performed as follows: at time $t=0$ the base is set impulsively to rotate with an angular velocity $\Omega=1 \mathrm{rads}^{-1}$. The solution is then allowed to saturate for $\operatorname{Re}=2680$, about $1.1 \%$ above the previously established limit for steady flow $[2,6]$. In this way the mode that causes the break from a steady state is isolated for investigation. Once a saturated 
periodic state is reached, defined as being when the relative peak-to-peak deviation over ten successive periods is less than $0.01 \%$, the flow is brought to a lower Reynolds number either via a step down or via a smooth (sinusoidal) ramp down. Both methods of decreasing Reynolds number were found to yield identical results. The flow is then allowed to evolve to a steady state as we monitored the decay of the instability mode via the time histories of flow parameters; we focus mostly on the axial component of velocity, although the choice is arbitrary. The non-linear analysis is performed using a truncated Landau model.

\subsection{The Landau equation}

While the behaviour of the bulk flow, governed by equations (1) and (2), is modeled using the SE method described in Section 2.1, we gain little insight into the instability dynamics directly from these simulations. Using the data generated via monitoring of flow variables in the SE simulations, in conjunction with a stability analysis model it is possible to analyse the transition behaviour directly. To that end we implement the non-linear Landau model, in order to help describe how the mode saturates. This is important in determining both the type of bifurcation and the amplitude of the mode beyond saturation. We also ascertain the linear growth rate which can be used, in much the same way as in a linear stability analysis, to predict transition points as a function of the control parameter (in this case the Reynolds number).

The Landau equation, as prescribed by Sheard et al. [13], used to model the non-linear growth and saturation of the instability is

$$
\frac{d A}{d t}=(\sigma+i \omega) A-l(1+i c)|A|^{2} A+\cdots,
$$

where $A(t)$ is a variable representing the amplitude of the perturbation. Modes that can be described by a cubic truncation of (3) exhibit non hysteretic behaviour through transition, depending on the polarity $( \pm)$ of $l$. If 
$l$ is positive, the transition is not hysteretic, if it is negative, hysteresis may occur, and higher order terms are required. The parameter $\sigma$ is the linear growth (or decay) rate of the instability. The model is described in detail by Provansal et al. [10] and Sheard et al. [13]. Following Sheard et al. [13], $A(t)$ is a complex oscillator written as $A=\rho e^{i \phi}$, and equation (3) is decomposed into real and imaginary parts

$$
\begin{aligned}
& \frac{d \log \rho}{d t}=\sigma-l \rho^{2}+\cdots, \\
& \frac{d \phi}{d t}=\omega-l c \rho^{2}+\cdots,
\end{aligned}
$$

where $\rho$ represents the magnitude of the perturbation and $\phi$ the phase. Given a time history of a quantity representing $A$, it is possible to calculate $d \log |A| / d t$ using finite differences from the envelope of the amplitude logarithm. From equation (4) a plot of this term against $|A|^{2}$ yields $\sigma$ and $l$ as the vertical axis intercept and the negative of the gradient, respectively.

In this fashion we proceed with the non-linear analysis of the transition from steady to unsteady flow in the axisymmetric subspace for an open cylinder with aspect ratio $H / R=1.5$.

\section{$3 \quad$ Results}

Figure 2(a) shows the growth and saturation of the envelope for the perturbation as manifest upon the axial component of the velocity at an arbitrary point in the flow. We use this parameter as a measure of the energy in the mode, that is $|A|=v_{\max }-v_{\min }$ over one period of the flow, for use in the Landau model, equation (4). The use of measurements taken at a single arbitrary location is valid due to the global nature of the instability under investigation [10]. A Reynolds number of $R e=2680$ was chosen as the saturated reference case from which the oscillations were allowed to decay to 



FiguRE 2: (a) The growth and saturation of the instability represented by the axial component of velocity, $v=u_{z}$, for $R e=2680$. (b) The derivative of the amplitude logarithm plotted against the square of the amplitude, for evolution from $R e=2680$ to $R e=2620$. The negative slope at small $|A|^{2}$ indicates an absence of hysteresis about the transition.

zero. The derivative of the amplitude logarithm is plotted against the square of the amplitude in Figure 2(b), for the reduction in Reynolds number from $\operatorname{Re}=2680$ to $R e=2620$. The primary points to note from Figure 2(b) are the linear trend and the negative slope, especially as $|A|^{2} \rightarrow 0$. This demonstrates that the transition is indeed a non hysteretic Hopf bifurcation where the unsteady branch makes a clean break from the steady branch, as predicted by Brons et al. [2], and also that equation (4) provides an accurate model of the amplitude behaviour in the transition region for the open cylinder flow. From the data presented in Figure 2(b) extrapolation to the axis at $|A|^{2}=0$ yields $\mathrm{d} \log |A| / \mathrm{dt}=\sigma$, the linear growth (or decay) rate. In this case a decay rate of $\sigma=-1.21 \times 10^{-3}$ is estimated.

Simulations were performed throughout the Reynolds number range $2600 \leq$ Re $\leq 2650$ and, from plots similar to Figure 2(b), decay rates were calcu- 
(a)



(b)

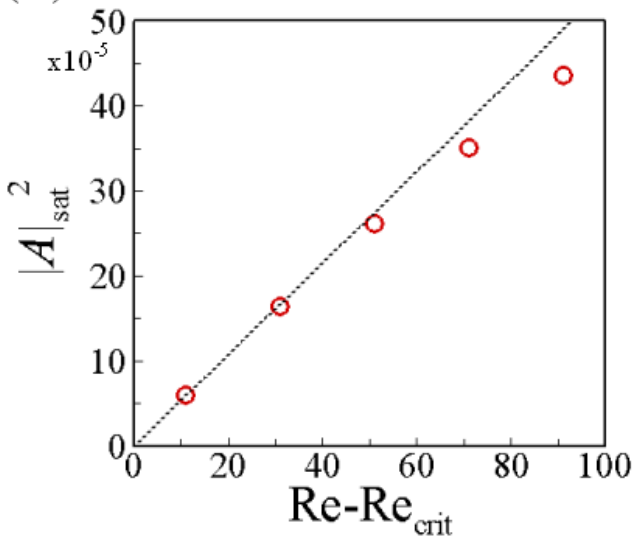

Figure 3: (a) The decay rate of the instability plotted against Reynolds number. The critical Reynolds number is found where $\sigma=0$. (b) The square of the post saturation velocity envelope amplitude plotted against Reynolds number, verifying the critical Reynolds number predicted by the Landau model in (a).

lated and are plotted in Figure 3(a) against Reynolds number. From a linear extrapolation to the point where $\sigma$ becomes positive the critical Reynolds number is estimated to be $\operatorname{Re}_{\text {crit }}=2659$. This value is within one percent agreement with the values reported by Lopez [6] and Brons et al. [2] of $\operatorname{Re}_{\text {crit }}=2650$ and 2640 , respectively.

An independent verification of this finding is possible via investigation of the strength of oscillations, after saturation, at Reynolds numbers beyond $\operatorname{Re}_{\text {crit }}$. Solving (4) for $d \log |\rho| / d t=0$ yields $\rho^{2}=|A|_{\text {sat }}^{2}=\sigma / l$, where $|A|_{\text {sat }}$ is the saturated oscillation amplitude. As shown in Figure 3(a), $\sigma$ is proportional to the Reynolds number increment above (and below) the critical Reynolds number $\left(\mathrm{Re}-\mathrm{Re}_{\text {crit }}\right)$ in the neighbourhood of a simple transition [13], and therefore $|A|_{\text {sat }}^{2} \propto \operatorname{Re}-\operatorname{Re}_{\text {crit }}$. Values of $|A|_{\text {sat }}^{2}$ measured at Re 
in the neighbourhood of the transition are shown in Figure 3(b). The linear trend in the vicinity of $R e-R_{\text {crit }}=0$ strongly supports the critical Reynolds number predicted by the Landau model. From the simulations performed at $R e>R_{\text {crit }}$ estimates of the period of the oscillation post transition were also obtained. For Reynolds numbers of $\mathrm{Re}=2680,2750$ and 3000 the dimensionless saturated periods, $\mathrm{T}^{*}=\mathrm{T} / \Omega$, were $\mathrm{T}^{*}=28.34,28.23$ and 27.86 , respectively, agreeing to better than one percent with corresponding values inferred from data presented by Lopez [6, Figure 10].

Figure 4 provides a visual presentation of the mode giving insight into its physical characteristics. It shows contours of the perturbation as manifest upon the azimuthal component of the vorticity. These were obtained by using the results from two SE simulations, subtracting the steady base flow field from a slightly perturbed flow field, in order to isolate the perturbation. One full period of the perturbation flow field is shown over nine frames at intervals of $T^{*} / 8$. A visualisation of this mode has not been previously published. Overlaid on the contours of Figure 4 are the axisymmetric steady-state streamlines of the base flow, showing the structure of the base flow in the meridional semi-plane. The mode is most intense at around 3/8th and $7 / 8$ th of the way through the cycle and from these frames it can be seen that, spatially, the instability is most energetic in the vicinity of the vortex breakdown bubble. Specifically, the maximum positive (negative) vorticity is observed at the outer (inner) edge of the interface between the vortex breakdown bubble and the primary meridional flow, with this situation reversing twice per period. We suggest that the cause of unsteadiness in this flow is an instability of the weak shear layer between the slow recirculation of the vortex breakdown bubble and the relatively fast primary circulation. 

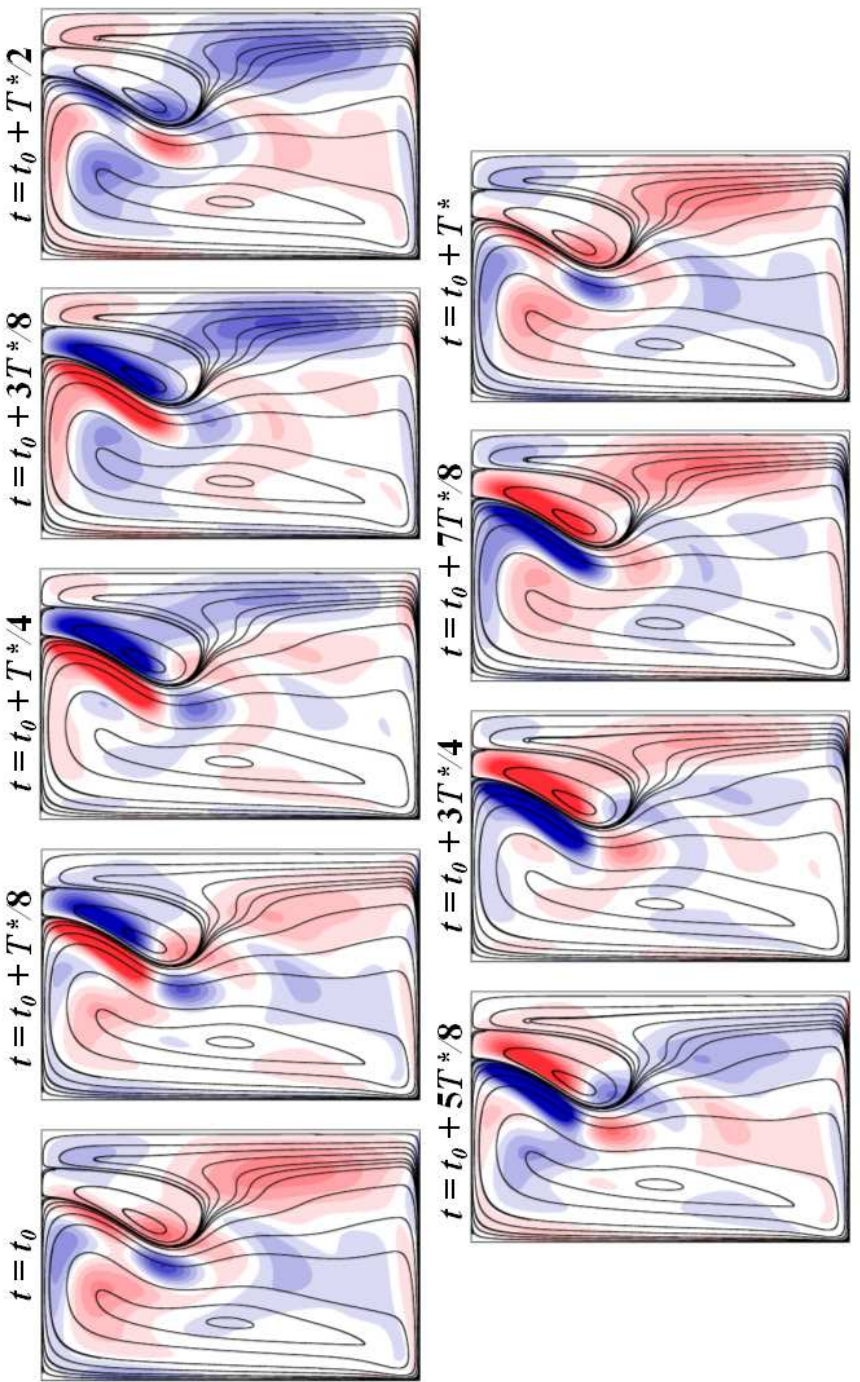

Figure 4: Velocity streamlines in the rz-plane overlaid on contours of the perturbation azimuthal vorticity, showing one period of the instability during the linear growth stage. Contours are spaced evenly about zero. 


\section{Conclusion}

We have employed axisymmetric simulations of the incompressible, time dependent, Navier-Stokes equations together with the non-linear Landau model to resolve a long standing discrepancy relating to swirling cylinder flows. It was confirmed that time dependence develops via a non hysteretic Hopf bifurcation. The transitional Reynolds number was predicted in agreement with previous findings and visualisations of the perturbation azimuthal vorticity in the linear regime provided hitherto uncovered insight into the cause of the transition. We suggest that the instability causing transition in this case is of the weak shear layer present at the interface between the primary meridional flow and the recirculating flow of the so called vortex breakdown bubble. As the focus of this article is the axisymmetric transition, it remains an open question as to whether the axisymmetric mode investigated here is the primary instability of the system. It has been shown to be the primary instability over a large range of the parameter space for the enclosed cylinder [3]. Whereas, it has been shown to be a secondary bifurcation in some open systems [8]. As the full range of aspect ratios has yet to be investigated for the free surface flow, further investigation of the bifurcation properties (including the breaking of symmetry) across a range of $\mathrm{H} / \mathrm{R}$ in open cylinder flows remains open and is the focus of a forthcoming article.

Acknowledgements Computations were aided by the Australian Partnership for Advanced Computing. S.J.C was supported by a Monash Departmental Scholarship. The authors thank Dr. Andreas Fouras for discussions helpful to the methodology of this study. 


\section{References}

[1] Blackburn, H. M. and Lopez, J. M., Symmetry breaking of the flow in a cylinder driven by a rotating end wall, Phys. Fluids, 12, 2000, 2698-2701. doi:10.1063/1.1313550 C252

[2] Brons, M., Voigt, L. K. and Sorensen, J. N., Topology of vortex breakdown bubbles in a cylinder with a rotating bottom and a free surface, J. Fluid Mech., 28, 2001, 133-148. http:

//journals . cambridge.org/action/displayAbstract?aid=65067 C253, C254, C255, C258, C259

[3] Gelfgat, A. Y., Bar-Yoseph, P. Z. and Solan, A., Three-dimensional instability of axisymmetric flow in a rotating lid-cylinder enclosure, J. Fluid Mech., 438, 2001, 363-377. doi:10.1017/S0022112001004566 $\mathrm{C} 252, \mathrm{C} 262$

[4] Hirsa, A. H., Lopez, J. M. and Miraghaie, R., Symmetry breaking to a rotating wave in a lid-driven cylinder with a free surface: Experimental observation, Phys. Fluids, 14, 2002, L29-L32. doi:10.1063/1.1471912 $\mathrm{C} 252$

[5] Karniadakis, G. E., Israeli, M. and Orszag, S. A., High-order splitting methods for the incompressible Navier-Stokes equations, J. Comp. Phys., 97, 1991, 414-443. doi:10.1016/0021-9991(91)90007-8 C255

[6] Lopez, J. M., Unsteady swirling flow in an enclosed cylinder with reflectional symmetry, Phys. Fluid, 7, 1995, 2700-2714. doi:10.1063/1.868717 C252, C253, C255, C259, C260

[7] Lopez, J. M., Cui, Y. D. and Lim, T. T., Experimental and numerical investigation of the competition between axizymmetric time-periodic modes in an enclosed swirling flow, Phys. Fluids, 18, 2006, 104106. doi:10.1063/1.2362782 C252 
[8] Lopez, J. M., Marques, F., Hirsa, A. H. and Miraghaie, R., Symmetry breaking in free-surface cylinder flows, J. Fluid Mech., 502, 2004, 99-126. doi:10.1017/S0022112003007481 C253, C262

[9] Marques, F. and Lopez, J. M., Precessing vortex breakdown mode in an enclosed cylinder flow, Phys. Fluids, 13, 2001, 1679-1682. doi:10.1063/1.1368849 C252

[10] Provansal, M., Mathis, C. and Boyer, L., Bénard-von Kármán instability: transient and forced regimes, J. Fluid Mech., 182, 1987, 1-22. doi:10.1017/S0022112087002222 C257

[11] Sheard, G. J., Leweke, T., Thompson, M. C. and Hourigan, K., Flow around an impulsively arrested circular cylinder, Phys. Fluids, 19, 2007, 083601. doi:10.1063/1.2754346 C254

[12] Sheard, G. J. and Ryan, K., Pressure-driven flow past spheres moving in a circular tube, J. Fluid Mech, 592, 2007, 233-262. doi:10.1017/S0022112007008543 C254

[13] Sheard, G. J., Thompson, M. C. and Hourigan, K., From spheres to circular cylinders: non-axisymmetric transitions in the flow fast rings, J. Fluid Mech., 506, 2004, 45-78. doi:10.1017/S0022112003006438 C256, C257, C259

\section{Author addresses}

1. S. J. Cogan, Fluids Laboratory for Aeronautical \& Industrial Research, Department of Mechanical \& Aerospace Engineering, Monash University 3800, Victoria, Australia. mailto: stuart.cogan@eng. monash. edu. au

2. G. J. Sheard, Fluids Laboratory for Aeronautical \& Industrial Research, Department of Mechanical \& Aerospace Engineering, Monash University 3800, Victoria, Australia. 
3. K. Ryan, Fluids Laboratory for Aeronautical \& Industrial Research, Department of Mechanical \& Aerospace Engineering, Monash University 3800, Victoria, Australia. 